\title{
Low Complexity Virtual Antenna Arrays using Cooperative Relay Selection
}

\author{
Aggelos Bletsas, Ashish Khisti, Moe Z. Win \\ Massachusetts Institute of Technology \\ 77 Massachusetts Ave, Cambridge, MA 02139 \\ aggelos@media.mit.edu, \{khisti,moewin\}@mit.edu
}

\begin{abstract}
We study the diversity-multiplexing tradeoff in cooperative diversity systems involving multiple relays. We focus on low complexity architectures that do not require simultaneous transmissions on the same frequency band and therefore are amenable to practical implementation with low-cost radios. We show that smart relay selection protocols achieve the same performance as previously proposed protocols that rely on multi-terminal space-time coding. Our study includes both analog and digital relays under a variety of relay selection criteria, irrespective of whether decision feedback is available in the network. Our results present an alternative to distributed space-time codes for realizing the potential gains in multiple relay cooperative systems and open new avenues for fruitful interaction between routing and cooperative diversity.
\end{abstract}

\section{INTRODUCTION}

Cooperative diversity is an attractive approach to improve performance in slow fading environments by creating distributed virtual antennas across different nodes in a wireless network. There has been a significant interest understanding the theoretical limits of such systems (e.g. [1], [2], [3], [4], [5]). Therefore, the study of practical architectures that achieve some of these limits is a fertile area of research [6].

A three node system with one source, one destination and one relay was investigated in [3] and generalized to the case of one source, one destination and multiple relays in [4]. The performance is characterized using the diversity-multiplexing tradeoff for multi-input-multioutput (MIMO) systems [7]. The proposed architecture in [4] requires that relays, successful in decoding the source transmission, help the destination by employing a distributed space-time code. While the performance of such systems increases monotonically with the number of relays, these architectures are perhaps not amenable to immediate practical implementation since they assume availability of distributed space-time codes across the relay nodes. While there has been some progress to- wards constructing space-time codes for classical MIMO systems (e.g. [8], [9]), cooperative systems involving two cooperating transmitters (e.g. [10]) and multiple relay systems involving global channel state information (CSI) knowledge (e.g. [11]), it remains to be seen how such constructions can be generalized in practical networks involving multiple relays with low-complexity radio front ends.

One architecturally appealing approach in multiple relay systems is relay selection. Based on end-to-end channel conditions, only one relay is used for cooperation. The system then reduces to the single-relay case for which several simple protocols exist. We show a rather surprising result that with a proper choice of relay selection criterion, there is in fact no performance loss in the sense of diversity-multiplexing tradeoff. While relay selection has been considered recently by other authors [12], [13] to the best of our knowledge our work is the first to compute the full diversity-multiplexing tradeoff of such systems and show that there is no loss compared to architectures based on distributed spacetime codes. We refer to our relay selection protocols as opportunistic relaying.

More recently it has been shown that simultaneous transmission across the source and relay node(s) can achieve better performance (e.g. [14],[5]. However our focus is on simple protocols which only need a single node to transmit at a given time, on a single frequency band. Such protocols are amenable to immediate implementation using existing low-cost radios. Accordingly, we compare our results with prior work, assuming orthogonal transmissions across all nodes [4], as a fair benchmark.

We note that cooperative transmission schemes require coordination overhead among the participating nodes. For example, the protocols in [5] require decode-andforward relays to know when all other relays have successfully decoded and retransmitted the message, 
with different but universally known codebooks. Such coordination (as envisioned in [5]) requires multiple beacon signals. In contrast, our schemes require relay selection only once, at an apriori given time point ${ }^{1}$ and therefore, network operation is simplified and coordination overhead is decreased.

We observe that our relay selection protocols achieve the same diversity-multiplexing tradeoff in [4] irrespective of whether the relay nodes perform amplify-andforward (AF) or decode-and-forward (DF). Further, we extend our result to the case where decision feedback is available from the destination and we observe additional gains that increase with the number of rounds of feedback. Our work sheds new insights into practical low-complexity architectures that combine relay selection and cooperative diversity, without sacrificing system performance.

In Section II we describe the channel model and present a wide variety of protocols in Section III. After presenting some useful Lemmas in Section IV, we present results for systems without feedback in Section V and for systems with feedback in Section VI.

\section{Channel Model}

In this section we describe the channel model under consideration. We consider a system with one source node, one destination node, and $M$ relay nodes. The channel gain between the source node and relay node $r$ is $a_{\mathrm{s} r}$, between the relay node $r$ and the destination is $a_{r \mathrm{~d}}$ between the source and destination is $a_{\mathrm{sd}}$. We assume that all channel gains are drawn i.i.d. $\mathcal{C N}(0,1)$ and remain constant throughout the course of transmission, as in the slow fading (quasi-static) channel model. When the source transmits a symbol $x_{\mathrm{s}}$, the received symbols at relay $r$ and the destination are given by:

$$
\begin{aligned}
& y_{r}=a_{\mathrm{s} r} x_{\mathrm{s}}+n_{r} \\
& y_{\mathrm{d}}=a_{\mathrm{sd}} x_{\mathrm{s}}+n_{\mathrm{d}}
\end{aligned}
$$

Here $n_{r}$ and $n_{\mathrm{d}}$ represent additive noise at the relay and destination respectively. We assume that these are complex Gaussian drawn independently from $\mathcal{C N}\left(0, N_{0}\right)$. Similarly we define the system equation for the link between relay $r$ and destination (with transmitted symbol $\left.x_{r}\right)$.

We shall assume an individual power constraint at each of the node. In particular $\mathbb{E}\left\{\left|x_{\mathrm{s}}\right|^{2}\right\} \leq P$ and $\mathbb{E}\left\{\left|x_{r}\right|^{2}\right\} \leq P$ for $r=1,2, \ldots, M$. Throughout we refer to $\rho \triangleq P / N_{0}$ as the average SNR on each link.

\footnotetext{
${ }^{1}$ Relay selection is done once for each coherence period.
}

Finally, note that in the high SNR regime of interest neither the individual power constraint nor the assumption of equal average SNR at each node are crucial towards our conclusions.

\section{Protocols With or without Feedback}

The protocols we consider have three main phases: (1) Distributed relay selection. (2) Transmission from the source and reception by the relay and the destination. (3) Transmission from the relay and reception by the destination. The three phases occur in the order as stated and over orthogonal channel uses.

a) Relay Selection: Throughout this work, we assume the existence of a distributed relay selection protocol that enables the selection of the "best" relay. We do not explicitly consider the overhead involved in the relay selection process. We refer the interested reader to our previous work [15], [16] that proposes such a protocol with minimal overhead and local channel knowledge.

Throughout this work, we assume that the relayselection is done before the message transmission from the source commences. For a discussion on selection schemes after the source transmission concludes please refer to the full paper [17]. We consider two possible objective functions in the process of selecting the best relay with channel gains $\left|a_{\mathrm{sb}}\right|$ and $\left|a_{\mathrm{bd}}\right|$ :

$$
\begin{aligned}
\min \left(\left|a_{\mathrm{sb}}\right|^{2},\left|a_{\mathrm{bd}}\right|^{2}\right) & =\max _{1 \leq r \leq M}\left\{\min \left(\left|a_{\mathrm{sr}}\right|^{2},\left|a_{\mathrm{rd}}\right|^{2}\right)\right\}(2) \\
\frac{2\left|a_{\mathrm{sb}}\right|^{2}\left|a_{\mathrm{bd}}\right|^{2}}{\left|a_{\mathrm{sb}}\right|^{2}+\left|a_{\mathrm{bd}}\right|^{2}} & =\max _{1 \leq r \leq M} \frac{2\left|a_{\mathrm{sr}}\right|^{2}\left|a_{r \mathrm{~d}}\right|^{2}}{\left|a_{\mathrm{sr}}\right|^{2}+\left|a_{r \mathrm{~d}}\right|^{2}}
\end{aligned}
$$

It turns out that both of these choices provide the same performance and hence are equivalent.

b) Transmission from Source and Relay: We next describe the source and relay transmission phases. The treatment of these phases differs depending on whether one allows feedback from the destination. In absence of any feedback, the source transmits over $n / 2$ channel uses and the relay over the remaining $n / 2$ channel uses. When a single-bit feedback is allowed, the destination broadcasts a NACK if it fails to decode after the source transmission. In this case the source transmits over $n$ channel uses and the relay, if it receives a NACK, transmits over the next $n$ channel uses. ${ }^{2}$ To emphasize the transmission over orthogonal channel uses, we number the transmitted symbols from the source as $x_{\mathrm{s}}[1], x_{\mathrm{s}}[2], \ldots, x_{\mathrm{s}}[t]$ and the transmitted symbols from the relay as $x_{r}[t+1], x_{r}[t+$

\footnotetext{
${ }^{2}$ This distinction in the codeword length will become clearer when we analyze the spectral efficiency of feedback protocols in Section VI.
} 
$2], \ldots, x_{r}[2 t]$. Here $t=n / 2$ for transmission in absence of feedback and $t=n$ if feedback is allowed. Finally we denote the received symbols from the source to destination as $y_{\mathrm{d}}[1], y_{\mathrm{d}}[2], \ldots y_{\mathrm{d}}[t]$ and from the relay to the destination as $y_{\mathrm{d}}[t+1], y_{\mathrm{d}}[t+2], \ldots y_{\mathrm{d}}[2 t]$. The received symbols at the relay are denoted as $y_{r}[1], y_{r}[2], \ldots y_{r}[t]$. The case of multiple round feedback is slightly different and treated in Section VI-B.

The source wishes to transmit one of $2^{n R}$ possible messages to the destination. We generically label the corresponding codewords as $c_{1}^{t}, c_{2}^{t}, \ldots, c_{2^{n R}}^{t}$, each referring to a length $t$ vector. Each codeword is sampled from i.i.d. Gaussian $\mathcal{N}(0, P)$ distribution. Thus we have $x_{s}[i]=c_{w}[i]$ for $i=1,2, \ldots, t$ where $w$ is the intended message. As discussed previously, each codeword is of length $t=n / 2$ in absence of feedback and of length $t=n$ if feedback is allowed. The received symbols at the relay and destination are given in eq. (1).

We consider both AF as well as DF protocols. In the AF scheme the best relay simply forwards its received symbol to the destination, appropriately scaling it to meet the power constraint i.e. $x_{r}[i+t]=\beta y_{r}[i]$ for $i=$ $1,2, \ldots t$, where $\beta=\sqrt{\frac{P}{N_{0}+\left|a_{b r}\right|^{2} P}}$. In the DF scheme, the relay attempts to decode the source transmission after $t$ channel uses. If it is successful in decoding the source transmission, it transmits the corresponding codeword $c_{w}^{t}$ to the destination. Otherwise it remains silent.

\section{USEFUL LEMMAS}

In this section, we provide several useful Lemmas which enable us to derive our results for the diversitymultiplexing tradeoff. The detailed proofs of these Lemmas can be found in [16],[17].

\section{Exponential Order}

The following Lemma provides the exponential order for the channel through the best-relay.

Lemma 1: The channel gains of the best relay selected either according to the min criterion (2) or the harmonic mean criterion (3) satisfies ${ }^{3}$,

$\mathbb{P}\left\{\left|a_{\mathrm{sb}}\right|^{2} \leq \rho^{-v}\right\}=\mathbb{P}\left\{\left|a_{\mathrm{bd}}\right|^{2} \leq \rho^{-v}\right\} \leq\left\{\begin{array}{l}\rho^{-M v} v \geq 0 \\ 1 \text { otherwise }\end{array}\right.$

The above Lemma essentially follows from the fact that the minimum of two exponentials of mean 1 is another exponential of mean $1 / 2$. Furthermore, the tail of maximum of $M$ exponentials essentially behaves as the

\footnotetext{
${ }^{3}$ The notation $f(\rho) \leq b(\rho)$ refers to $\lim _{\rho \rightarrow \infty} \frac{\log f(\rho)}{\log b(\rho)} \leq 1$. The notation of $\doteq$ is defined similarly.
}

tail of the sum of $M$ exponentials. This observation is key in establishing that our opportunistic relay selection algorithm has the same performance as a scheme where all the potential $M$ relays transmit using a distributed space-time code.

Lemma 2: With $f(x, y)=\frac{x y}{x+y+1}$ we have that

$$
\begin{aligned}
& \operatorname{Pr}\left(f(\rho a, \rho b) \leq \rho^{2 r}\right) \leq \\
& \operatorname{Pr}\left(\min (a, b) \leq \rho^{2 r-1}+\rho^{r-1} \sqrt{1+\rho^{2 r}}\right) .
\end{aligned}
$$

The above Lemma is used to establish that the AF scheme has the same performance as the DF scheme under opportunistic relaying.

We now present detailed analysis of all the protocols described in Section III.

\section{Virtual ArRays with Zero-FeEdback}

In this section, we consider the case of no feedback. In $\mathrm{AF}$, the source transmits over $n / 2$ channel uses and the relay subsequently transmits over $n / 2$ channel uses. The destination attempts to decode after $n$ channel uses. The destination fails to decode under the following event:

$$
\begin{aligned}
& \mathcal{E}_{\mathrm{AF}} \triangleq \\
& \left\{I_{\mathrm{AF}}=\frac{n}{2} \log \left(1+\rho\left|a_{\mathrm{sd}}\right|^{2}+f\left(\rho\left|a_{\mathrm{sb}}\right|^{2}, \rho\left|a_{\mathrm{bd}}\right|^{2}\right)\right) \leq n R\right\}
\end{aligned}
$$

here the function $f$ is defined as in Lemma 2.

In $\mathrm{DF}$, the best relay attempts to decode the message. The relays fails to decode the message under the following event:

$$
\mathcal{E}_{\mathrm{DF}}^{\mathrm{R}} \triangleq\left\{I_{\mathrm{DF}}^{\mathrm{R}}=\frac{n}{2} \log \left(1+\left|a_{\mathrm{sb}}\right|^{2}\right)<n R\right\} .
$$

The destination fails to decode the message in DF when $\mathcal{E}_{\mathrm{DF}} \triangleq\left\{I_{\mathrm{DF}} \leq n R\right\}$, where

$$
I_{D F}=\left\{\begin{array}{l}
\frac{n}{2} \log \left(1+\rho\left|a_{\mathrm{sd}}\right|^{2}\right) \text { relay can't decode } \\
\frac{n}{2} \log \left(1+\rho\left|a_{\mathrm{sd}}\right|^{2}+\rho\left|a_{\mathrm{bd}}\right|^{2}\right) \text { relay decodes }
\end{array}\right.
$$

Using the results of Lemma 1 and 2, the above outage events can be analyzed and the following result is shown in [16]:

Theorem 1: The diversity-multiplexing gain of zerofeedback opportunistic (DF as well as AF) relaying is:

$$
d(r)=(M+1)(1-2 r) .
$$

Note that the diversity-multiplexing tradeoff in the above Theorem is same as that for the $M$ relay case studied by Laneman [4], using space-time codes. Our result 
shows that one can get essentially the same performance via opportunistic relaying and using either analog or digital relays. Thus the gains from user cooperation arise fundamentally from the existence of multiple paths rather than the use of distributed space-time codes. Note that there has been a lot of interest in relay selection algorithms for cooperative communications. To the best of our knowledge, our result is the first one that makes a precise statement regarding the diversity-multiplexing performance optimality of relay selection protocols.

\section{Virtual ARrays with Single-Bit FeEdback}

\section{A. Single Round of Feedback}

We now present the results for the case when the destination attempts to decode the source transmission and sends a single-bit indicating whether the reception was successful. The relay transmits only when the destination fails to decode from the source transmission.

An outage event occurs if the destination fails to decode after the second attempt. This happens when the mutual information is less than $n R$. In the AF case, the mutual information is given by:

$$
I_{A F F}=n \log \left(1+\rho\left|a_{\mathrm{sd}}\right|^{2}+f\left(\rho\left|a_{\mathrm{bd}}\right|^{2}, \rho\left|a_{\mathrm{sb}}\right|^{2}\right)\right)
$$

Similarly in the DF protocol, an outage event occurs if mutual information given by the following expression is less than $n R$ :

$$
\begin{aligned}
& I_{D F F}= \\
& =\left\{\begin{array}{l}
n \log \left(1+\rho\left|a_{\mathrm{sd}}\right|^{2}\right) \text { relay can't decode } \\
n \log \left(1+\rho\left|a_{\mathrm{sd}}\right|^{2}+\rho\left|a_{\mathrm{bd}}\right|^{2}\right) \text { relay decodes }
\end{array}\right.
\end{aligned}
$$

In the above definition, the event that the relay cannot decode is given by

$$
\mathcal{E}_{\mathrm{DFF}}^{\mathrm{R}} \triangleq\left\{I_{\mathrm{DFF}}^{\mathrm{R}}=n \log \left(1+\rho\left|a_{\mathrm{sb}}\right|^{2}\right)<n R\right\} .
$$

Note that in these protocols, the best relay transmits only if the destination is not successful in decoding the message. Accordingly, the spectral efficiency of the protocol is a variable quantity. In particular, the average spectral efficiency is given by:

$$
\bar{R}=R \mathbb{P}\left\{\overline{\mathcal{E}_{1}}\right\}+\frac{R}{2} \mathbb{P}\left\{\mathcal{E}_{1}\right\}
$$

We now present the analysis of outage probability for opportunistic relaying.

Lemma 3: Let $\mathcal{O}$ ut denote the outage event in the DF protocol with single-bit feedback (with mutual information given in eq. (10)). Then with $r \doteq R / \log \rho$, we have $\mathbb{P}\{\mathcal{O} u t\} \leq \rho^{-(M+1)(1-r)}$.
First note that:

$$
\begin{aligned}
& \mathbb{P}\left\{\mathcal{O} \text { ut } \mid \mathcal{E}_{\mathrm{DFF}}^{\mathrm{R}}\right\}=\mathbb{P}\left\{\left|a_{\mathrm{sd}}\right|^{2}<\frac{2^{R}-1}{\rho}\right\} \leq \rho^{r-1} \\
& \mathbb{P}\left\{\mathcal{E}_{\mathrm{DFF}}^{\mathrm{R}}\right\}=\mathbb{P}\left\{\left|a_{\mathrm{sb}}\right|^{2}<\frac{2^{R}-1}{\rho}\right\} \dot{\leq} \rho^{M(r-1)}
\end{aligned}
$$

Where we used the result in Lemma 1 in the (14). Similarly, we have

$$
\begin{aligned}
& \mathbb{P}\left\{\text { Out } \mid \overline{\mathcal{E}_{\mathrm{DFF}}^{\mathrm{R}}}\right\} \\
\leq & \mathbb{P}\left\{\left|a_{\mathrm{sd}}\right|^{2}<\frac{2^{R}-1}{\rho} \bigcap\left|a_{\mathrm{bd}}\right|^{2}<\frac{2^{R}-1}{\rho}\right\} \\
\leq & \rho^{(1+M)(r-1)} \\
& \mathbb{P}\left\{\overline{\mathcal{E}_{\mathrm{DFF}}^{\mathrm{R}}}\right\} \leq 1
\end{aligned}
$$

and

The result of the Lemma follows from (13)-(16).

We next present a similar Lemma for the AF protocol.

Lemma 4: Let $O$ ut denote the outage event in the $\mathrm{AF}$ protocol with single-bit feedback (for which the mutual information is given in eq. (9)). Then with $r \doteq R / \log \rho$, we have $\mathbb{P}\{\mathcal{O} u t\} \dot{\leq} \rho^{-(M+1)(1-r)}$.

To prove the above Lemma, we define the following events:

$$
\begin{aligned}
& A \equiv\left\{\log _{2}\left(1+\left|a_{\mathrm{sd}}\right|^{2} \rho\right)<R\right\} \\
& B \equiv\left\{\log _{2}\left(1+\left|a_{\mathrm{sd}}\right|^{2} \rho+f\left(\left|a_{\mathrm{sb}}\right|^{2} \rho,\left|a_{\mathrm{bd}}\right|^{2} \rho\right)\right)<R\right\}
\end{aligned}
$$

Note that an outage event happens if the direct link from source to destination is weak (i.e. event A occurs) and the link via the relay is weak (i.e. event B occurs). 
Accordingly,

$$
\begin{aligned}
& \operatorname{Pr}\{\mathcal{O} u t\}=\operatorname{Pr}\{A \cap B\} \\
& =\operatorname{Pr}\left\{\left|a_{\mathrm{sd}}\right|^{2}<\frac{2^{R}-1}{\rho} \bigcap\right. \\
& \left.\quad\left|a_{\mathrm{sd}}\right|^{2}+\frac{1}{\rho} f\left(\left|a_{\mathrm{sb}}\right|^{2} \rho,\left|a_{\mathrm{bd}}\right|^{2} \rho\right)<\frac{2^{R}-1}{\rho}\right\} \\
& =\operatorname{Pr}\left\{\left|a_{\mathrm{sd}}\right|^{2}<\frac{2^{R}-1}{\rho}\right\} \times \\
& \qquad \operatorname{Pr}\left\{f\left(\left|a_{\mathrm{sb}}\right|^{2} \rho,\left|a_{\mathrm{bd}}\right|^{2} \rho\right)<\left(2^{R}-1\right)\right\} \\
& \dot{\operatorname{Pr}}\left\{\left|a_{\mathrm{sd}}\right|^{2}<\rho^{r-1}\right\} \operatorname{Pr}\left\{f\left(\left|a_{\mathrm{sb}}\right|^{2} \rho,\left|a_{\mathrm{bd}}\right|^{2} \rho\right)<\rho^{r}\right\} \\
& \leq \operatorname{Pr}\left\{\left|a_{\mathrm{sd}}\right|^{2}<\rho^{r-1}\right\} \times \\
& \operatorname{Pr}\left\{\min \left\{\left|a_{\mathrm{sb}}\right|^{2},\left|a_{\mathrm{bd}}\right|^{2}\right\}<\rho^{r-1}+\rho^{0.5 r-1} \sqrt{1+\rho^{r}}\right\} \\
& \dot{\leq} \rho^{r-1} \rho^{M(r-1)}=\rho^{(M+1)(r-1)}
\end{aligned}
$$

In the two last steps, we used Lemma 2, the fact that $\rho^{r-1} \sqrt{1+\rho^{2 r}} \rightarrow \rho^{2 r-1}$ as $\rho \rightarrow \infty$.

Recall from (12) that $R$ is not the true spectral efficiency of the system. However the next lemma shows that it is indeed very close to the true spectral efficiency $\bar{R}$.

$$
\begin{aligned}
& \text { Lemma 5: } \bar{R} \doteq R \\
& \quad \text { Proof: } \\
& \bar{R}=R \mathbb{P}\left\{\left|a_{\mathrm{sd}}\right|^{2} \geq \rho^{r-1}\right\}+\frac{R}{2} \mathbb{P}\left\{\left|a_{\mathrm{sd}}\right|^{2} \leq \rho^{r-1}\right\} \\
& \quad \doteq R\left(1-\rho^{r-1}\right)+\frac{R}{2} \rho^{r-1}=R\left(1-\frac{1}{2} \rho^{r-1}\right) \\
& \quad \doteq R
\end{aligned}
$$

The following Theorem is a direct consequence of Lemma 3, 4 and 5.

Theorem 2: The diversity-multiplexing tradeoff of both DF and AF protocols with a single-bit feedback is given by $d(r)=(M+1)(1-r)$.

We note that single-bit/single-round feedback can provide the performance of a $(M+1) \times 1$ MISO system, which is substantially better than the case without feedback in Theorem 1. This is because, in the high SNR regime, the destination will decode the direct source transmission most of the time. The relay needs to transmit for a very small fraction of the time. Hence the effective multiplexing gain is the same as that of the direct transmission. On the other hand, the failure event occurs only if the channel links from source to relay and the destination are both in outage. We note that a similar effect has been observed in [3] for the three node channel and in [18] for the MIMO ARQ channel.

\section{B. Multiple Rounds of Feedback}

So far our discussion has been limited to a single round of feedback. An outage event is declared if the destination cannot decode after the relay transmission. A natural generalization of this scheme is to allow the destination send a NACK packet if it fails to decode after the relay transmission and have a total of $L$ "rounds" of transmission. We define one round of transmission as consisting of $2 n$ channel uses with the source transmitting over $n$ channel uses and the relay transmitting over $n$ channel uses. Furthermore we assume that the source uses an independent codebook for each round of transmission. Thus the codewords in round $j$ are denoted by $c_{1}^{n}(j), c_{2}^{n}(j), \ldots, c_{w}^{n}(j)$. An outage event in the AF scheme after $L$ rounds of feedback is given by a straightforward extension of (9).

$$
\mathcal{E}_{2}^{L} \triangleq\left\{n L \log \left(1+\rho\left|a_{\mathrm{sd}}\right|^{2}+f\left(\rho\left|a_{\mathrm{bd}}\right|^{2}, \rho\left|a_{\mathrm{sb}}\right|^{2}\right)\right) \leq n R\right\}
$$

Note that the mutual information gets amplified by a factor of $L$ if $L$ rounds of transmission are allowed. By using essentially the same argument in Lemma 5, we note that $\bar{R} \doteq R$. Thus, we note that the diversitymultiplexing curve is scaled by a factor of $L$ horizontally. Analogous reasoning for the DF protocol is under investigation.

Theorem 3: The achievable diversity-multiplexing tradeoff for AF protocols with $L$ rounds of feedback (i.e. $2 \mathrm{~L}-1$ NACKs) is given by $d(r)=(M+1)\left(1-\frac{r}{L}\right)$ for $0 \leq r \leq 1$.

Note that our result shows that the performance improves significantly with multiple rounds of feedback. A similar result has been observed for the MISO systems in [18] and for cooperative diversity systems employing dynamic DF strategies in [19].

\section{CONCLUSION}

We explored the fruitful interaction between relay selection (which can be viewed as a routing protocol) and cooperative diversity (which is a physical layer protocol). We showed that a smart relay selection algorithm provides an alternative to using distributed space-time codes in cooperative diversity systems. In particular, with 


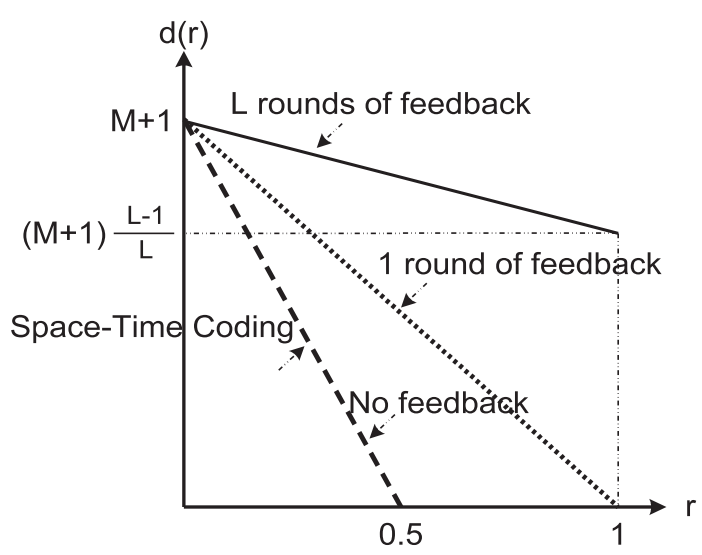

Fig. 1. The diversity-multiplexing gain tradeoff of the examined protocols. Opportunistic relaying without feedback achieves the same tradeoff curve as space-time coding, opportunistic relaying and single round of feedback achieves the MISO curve (without feedback) and opportunistic relaying with $L$ rounds of feedback achieves further improved performance.

a smart relay selection algorithm, one needs to only consider coding for a three node setup while achieving the theoretical performance of a $M$ node system, employing distributed space-time codes. Our simplification makes the system amenable to immediate hardware implementation and one such implementation has been reported in [6]. In addition, we explored the role of feedback and showed dramatic performance improvement with just a single-bit of feedback. In fact the performance is same as that of a $(M+1) \times 1$ MISO system that does not employ feedback. We also observed that further improvement was possible if additional rounds of feedback were allowed.

We hope that our work sparks further interest in implementation of distributed relay selection protocols. While one such protocol based on distributed timers has been proposed in [15] and the experimental results have been reported in [6], we believe this is a very fertile area for further innovation. On the theoretical side we note that our focus was on orthogonal protocols i.e., protocols where the source and relay transmit over orthogonal channel uses. It would be interesting to consider simplification of non-orthogonal protocols such as those proposed in [5] using relay selection techniques.

\section{REFERENCES}

[1] J. H. Winters, "On the capacity of radio communication systems with diversity in rayleigh fading environment," IEEE J. Select. Areas Commun., vol. 5, no. 5, p. 871878, June 1987.

[2] A. Sendonaris, E. Erkip, and B. Aazhang, "User cooperation diversity-Part I: System description," IEEE Trans. Commun., vol. 51, no. 11, pp. 1927-1938, Nov. 2003.
[3] J. N. Laneman, D. N. C. Tse, and G. W. Wornell, "Cooperative diversity in wireless networks: Efficient protocols and outage behavior," IEEE Trans. Inform. Theory, vol. 50, no. 12, pp. 3062-3080, Dec. 2004.

[4] J. N. Laneman and G. W. Wornell, "Distributed space-time coded protocols for exploiting cooperative diversity in wireless networks," IEEE Trans. Inform. Theory, vol. 59, pp. 2415-2525, Oct. 2003.

[5] K. Azarian, H. E. Gamal, and P. Schniter, "On the achievable diversity-vs-multiplexing tradeoff in cooperative channels," IEEE Trans. Inform. Theory, vol. 51, pp. 4152-4172, Dec. 2005.

[6] A. Bletsas, "Intelligent antenna sharing in cooperative diversity wireless networks," Ph.D. dissertation, Massachusetts Institute of Technology, Cambridge, MA, Sept. 2005.

[7] L. Zheng and D. N. C. Tse, "Diversity and Multiplexing: A Fundamental Tradeoff in Multiple Antenna channels," IEEE Trans. Inform. Theory, vol. 49, pp. 1073-1096, May 2003.

[8] H. E. Gamal, G. Caire, and M. O. Damen, "Lattice coding and decoding achieve the optimal diversity-multiplexing tradeoff of mimo channels," IEEE Trans. Inform. Theory, vol. 50, no. 6, pp. 968-985, June 2004.

[9] K. Lu, S. Fu, and X.-G. Xia, "Closed form designs of complex orthogonal space-time block codes of rates $(k+1) /(2 k)$ for $2 k-1$ or $2 \mathrm{k}$ transmit antennas," IEEE Trans. Inform. Theory, 2006, to be published.

[10] M. Dohler, E. Lefranc, and H. Aghvami, "Space-time block codes for virtual antenna arrays," in PIMRC, Lisbon, Portugal, 2002.

[11] Y. Jing and B. Hassibi, "Distributed space-time coding in wireless relay networks-part i: basic diversity results," IEEE Trans. Wireless Commun., 2005, submitted.

[12] B. Zhao and M. C. Valenti, "Practical relay networks: A generalization of hybrid-arq," IEEE J. Select. Areas Commun., (Special Issue on Wireless Ad Hoc Networks), vol. 23, no. 1, pp. 7-18, Jan. 2005.

[13] J. Luo, R. S. Blum, L. J. Cimini, L. J. Greenstein, and A. M. Haimovich, "Link-failure probabilities for practical cooperative relay networks," in Proceedings of IEEE VTC, June 2005.

[14] R. U. Nabar, H. Blcskei, and F. W. Kneubhler, "Fading relay channels: Performance limits and space-time signal design," IEEE J. Select. Areas Commun., vol. 22, no. 6, pp. 1099-1109, Aug. 2004.

[15] A. Bletsas, A. Lippman, and D. P. Reed, "A simple distributed method for relay selection in cooperative diversity wireless networks, based on reciprocity and channel measurements," in Proceedings of IEEE 61st VTC, Stockholm, Sweden, May-Jun. 2005.

[16] A. Bletsas, A. Khisti, D. P. Reed, and A. Lippman, "A simple cooperative diversity method based on network path selection," IEEE J. Select. Areas Commun., 2006, to be published.

[17] A. Bletsas, A. Khisti, and M. Z. Win, "Select and forward: Unifying cooperative diversity and routing in a common framework," 2006, in preparation.

[18] H. E. Gamal, G. Caire, and M. O. Damen, "The mimo arq channel," IEEE Trans. Inform. Theory, Dec. 2005, submitted.

[19] K. Azarian, H. E. Gamal, and P. Schniter, "On the achievable diversity-multiplexing tradeoff in half duplex cooperative channels," in Proc. Asilomar Conf. on Signals, Systems and Computers, Monticello, IL, Oct. 2004. 